\section{A Rare Coronary Anomaly: Double Right}

Okan Gulel, MD, et al.: Clin Cardiol 2007;30:309

\section{To the Editor:}

The paper by Gulel et al. ${ }^{1}$ in the Journal provokes a response by an author, who has dedicated a great deal of effort to establishing a discipline in the chapter of coronary anomalies.

The above named authors ${ }^{1}$ state that they report a "rare" anomaly, featuring double right coronary artery (RCA). I contend that the entity they describe is actually neither rare (indeed, it is the most frequent of coronary anomalies, being $1.23 \%$ of a continuous series of a general population, if one uses defined criteria of normality and anomalies and performs prospective studies), ${ }^{2}$ nor is it a case of two RCAs. The case is indeed one of "split right coronary artery."

Let me explain why it is important (if we want to make sense of reporting unusual anatomy) to establish some principles and definitions in this discipline, before venturing into the field.

Principle \#1: a coronary artery is essentially defined by the dependent territory. ${ }^{2}$ In the proposed case, the two branches originated at the right sinus of Valsalva single ostium and go to the posterior/inferior wall of the left ventricle, a territory that could be either of the RCA (which would be called dominant, in such case) or of the circumflex (which would be called dominant, in such case). Since both coronary branches originated from the right sinus of
Valsalva, the two should be considered part of a dominant RCA: the unusual feature was the presence of two separate branches supplying the posterior descending territory. The splitting is proximal, in such example, and it leads to one branch that lies, as usual, in the right-sided atrioventricular (AV) groove, and leads to the upper (basal) portion of the posterior descending, and one branch that courses over the right ventricular free wall, and then it supplies the more distal posterior descending artery; there is no other coronary artery (double), but two in place of one proximal conductive artery. The essential splitting is that of the posterior descending, in fact.

As the authors noted, coronary arteriosclerotic changes were present, and angiographically, they seemed to only affect the atrioventricular (or basal) RCA branch, which is the most frequent case, in such forms. The cause is unknown, but likely related to the higher amount of fat in the $\mathrm{AV}$ sulcus, with respect to the right ventricular branch: but this interpretation is speculative.

Paolo Angelini, MD

Texas Heart Institute; Baylor College of Medicine, Houston, Texas, USA

\section{References}

1. Gulel O, Durna K, Demircan S, Yazici M: A rare coronary anomaly: double right coronary artery. Clin Cardiol 2007;30:309

2. Angelini P, Villason S, Chan AV, Diez JG: Normal and anomalous coronary arteries in humans. In Coronary Artery Anomalies. A Comprehensive Approach (Ed. Angelini P). Philadelphia, PA: Lippincott Williams and Wilkins, 1999; pp 27-151 